\title{
I Kissed Homework Goodbye: Replacing Homework with Online Quizzes
}

\section{Dr. Thomas S. Soerens, Messiah College}

Thomas Soerens is Professor of Engineering at Messiah College. He teaches in Civil and Environmental engineering with specialization in storm water, ground water, and water treatment. He performs design and applied research in water systems in rural developing communities. He is a Professional Engineer and a Certified Ecological Designer. 


\section{Kissed Homework Goodbye: Using Online Quizzes in Place of Homework}

\section{Abstract}

Do online quizzes with immediate feedback promote better student learning than homework assignments for upper level engineering classes? A junior-level Environmental Engineering class was reworked to use multiple-choice quizzes in a learning management system rather than paper homework. Similar approaches have shown encouraging improvement in student motivation [1], in providing feedback to students [2] and in exam performance [3]. An objective for using this approach in Environmental Engineering was to allow the students to receive immediate feedback on their skills. For example, in calculating the $\mathrm{pH}$ of a composite water sample (with some simplifying assumptions), there are common mistakes that students make that will lead them to specific incorrect answers. When they submit their answers for the quiz, the quiz is set up to tell them why they got that wrong answer. Then they make retake the quiz. In addition to the problem-based quizzes, reading-based quizzes with questions based on the assigned reading were used. For some readings, students were assigned to participate in discussion forums where students could see others' writings and are encouraged to engage with and add to others' writings. There were a few assignments that did not lend themselves to quizzes. For those, students uploaded a spreadsheet solution to Canvas.

Learning improvement was measured by a 50 question multiple-choice final exam, which was identical to the final in the previous offering. The average score improved from $85.5 \%$ in the previous offering to $87.9 \%$ in the new model. In previous years, problems on reaction kinetics and on chemistry were frequently answered incorrectly and a primary motivation for the new system was to correct this with quizzes that offered feedback. With the new model, the students performed $100 \%$ correctly on the five reaction kinetics problems and $86 \%$ on the five chemistry questions on the final exam. However, students did poorly on a landfill sizing problem that was similar to a quiz and did poorly on a TMDL question that was only covered in a discussion forum. Students also performed poorly on a problem that was only covered in a lab exercise.

Scores on the quizzes themselves were quite high and were not very useful for assessing individual student performance. Feedback from the students on the quiz approach was requested with a survey and overall reaction was positive and encouraging. A drawback of the online quizzes it that it is easier to copy another student's quiz answers than it is to copy a paper homework. This paper presents observations and assessments of the course.

\section{Introduction}

Engineering homework problems are assigned to give students practice doing problems that involve the skills and outcomes of the course and to assess their performance of these problems. For this Environmental Engineering class in the past, the homework solutions were posted on the 
afternoon the homework was collected, but the grading and returning the papers to the students often lagged. By using online quizzes instead of homework, students can receive immediate feedback, can make another attempt at the problem, and do not have to wait for the instructor to grade and return the homework.

Schmidt et al. [1] found that using quizzes in place of homework can increase student motivation. Brunnhoeffer [2] found that a similar approach keeps the students up to date with where they stand in the course. Dufresne et al. [3] found that web-based homework offerings led to statistically significant higher overall exam performance. In the Environmental Engineering class, common mistakes on certain types of problems and on the corresponding exams reoccur every class offering. The Environmental Engineering class is a good candidate course for substituting online quizzes for paper homework to increase motivation, provide quicker feedback and grading, and improve exam performance.

The goals of this study were to

1. Provide immediate feedback to students on problem-solving

2. Reduce grading time for the instructor

3. Reduce the lag between when a student does the homework and when they receive their score (keep students up to date)

4. Increase student motivation

5. Improve exam performance, especially in previously low-scoring topic areas

Assessment of the study was done by final exam problem scores in areas related to the quizzes and by a student survey related to the quizzes.

\section{Quiz examples}

Below are examples of a multiple choice questions related to $\mathrm{pH}$. Those familiar with water chemistry will know that with buffering and other processes, the $\mathrm{pH}$ of those samples mixed together is not quite that predictable, but this fits with how $\mathrm{pH}$ has been presented at this point in the course.

The text comments below the answers in the quiz are shown to the students after they submit the quiz. For some questions, the solution method is shown if they choose correctly. Figures 1 and 2 show questions related to $\mathrm{pH}$. Figure 2 shows an example where the quiz problem was a problem from the textbook: 


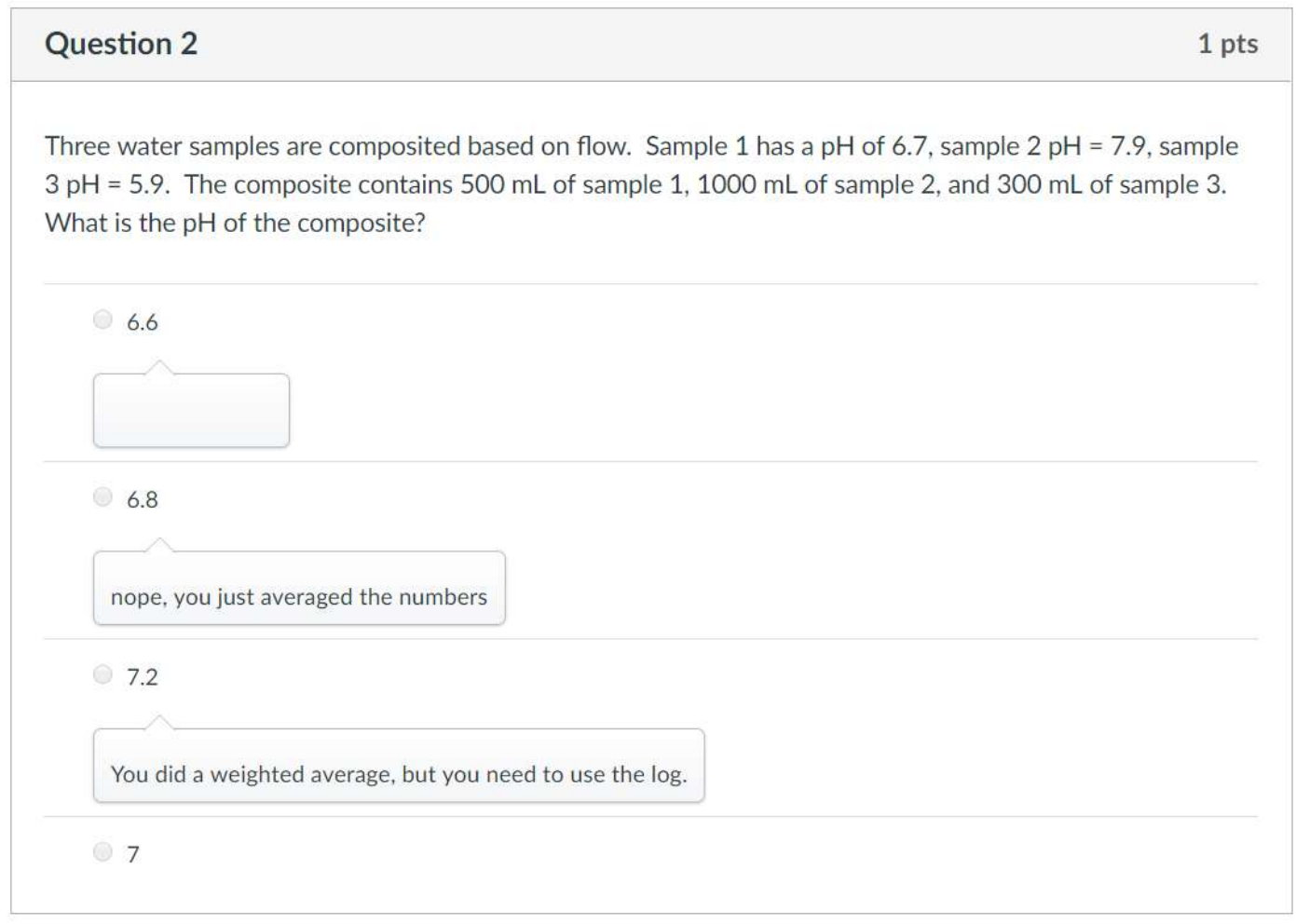

Figure 1. Example Quiz Question with the responses the students see based on their choice

\begin{tabular}{|l|l|}
\hline Question 5 & 1 pts \\
Text problem 3.18 \\
(hint: see example 3.8) \\
pH $=9.7$ \\
that's $\mathrm{pOH}$, you need $\mathrm{pH}$ \\
$\mathrm{pH}=8.3$ \\
It's $2 \mathrm{OH}$-, so you need the square root and then convert from pOH to $\mathrm{pH}$ \\
$\mathrm{pH}=4.8$ \\
It's 2 OH-, so you need the square root
\end{tabular}

Figure 2. Example quiz question using text problem 
The learning management system allows the instructor to see what each student answered in each attempt, the time they took, and related statistics. The students can read the problems ahead of time and work on the solutions before taking the quiz. Perhaps it would be better for learning assessment if they did not see the problems first and had a limit to the time, but the goal was to match the homework environment where they can take time, refer to their texts and their friends, and then finalize the solution. This approach, however, lead to high quiz scores that were not that useful for individual assessment and tended to bias the overall course grades upward.

The other type of online substitution for homework was discussion forums to discuss the assigned readings from the text an online. The online discussion where students are encouraged or required to react to and interact with the previous responses have proven to be interesting and valuable in other courses in the department and may be a good fit for Environmental Engineering. Some of the discussions are compiling facts and some are more interactive and subjective. Below is an example of the prompt for a discussion.

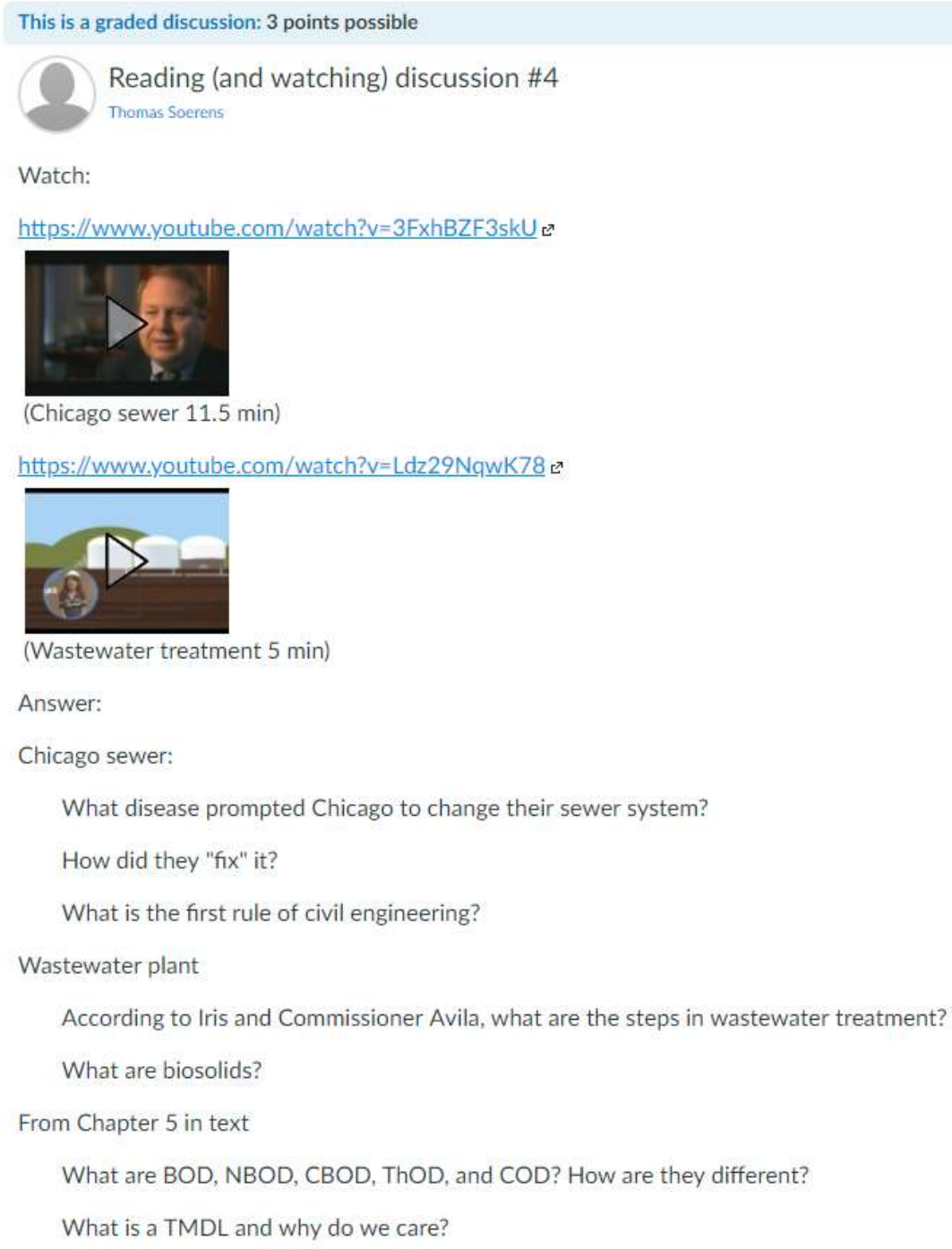

Figure 3 Online discussion prompt for introducing wastewater 


\section{Results and feedback}

Learning improvement was measured by a 50 question multiple-choice final exam, which was identical to the final in the previous offering. The average final exam score improved from $85.5 \%$ in the previous offering to $87.9 \%$ in the new model. Poor performance on the kinetic and chemistry problems on past final exams was a motivation to try this new approach. With the new model, the students performed $100 \%$ correctly on the five reaction kinetics problems and $86 \%$ (percentage of students choosing correct answer) on the five chemistry questions on the final exam. Individual question scores from the previous year were not saved -- the idea and decision to move to the online quiz approach was made between offerings -- but this is perceived as a significant improvement.

Improvements were not seen in every area, however. Students did poorly on a landfill sizing problem that was similar material to a quiz, but in a different format, even though they scored well on the quiz. Students also did poorly on a TMDL question that was only covered in a discussion forum. And students performed poorly on a hardness problem that was only covered in a lab exercise. The landfill problem result emphasizes the need to explore this in more detail in class. The other two questions emphasize that testing in a quiz or homework during the semester improves student performance on those questions on the final.

In addition to Chemistry, Kinetics, and Solid Waste, the topics covered in other quizzes were BOD, Risk Assessment, Air Pollution, Sedimentation, Wastewater Processes, and Units and Quantities. Students scored at least $87 \%$ on the final exam in questions related to these other areas; however, these other areas were not low-scoring areas in the past. Figure 4 shows the scores of the quizzes and of the corresponding final exam problems.

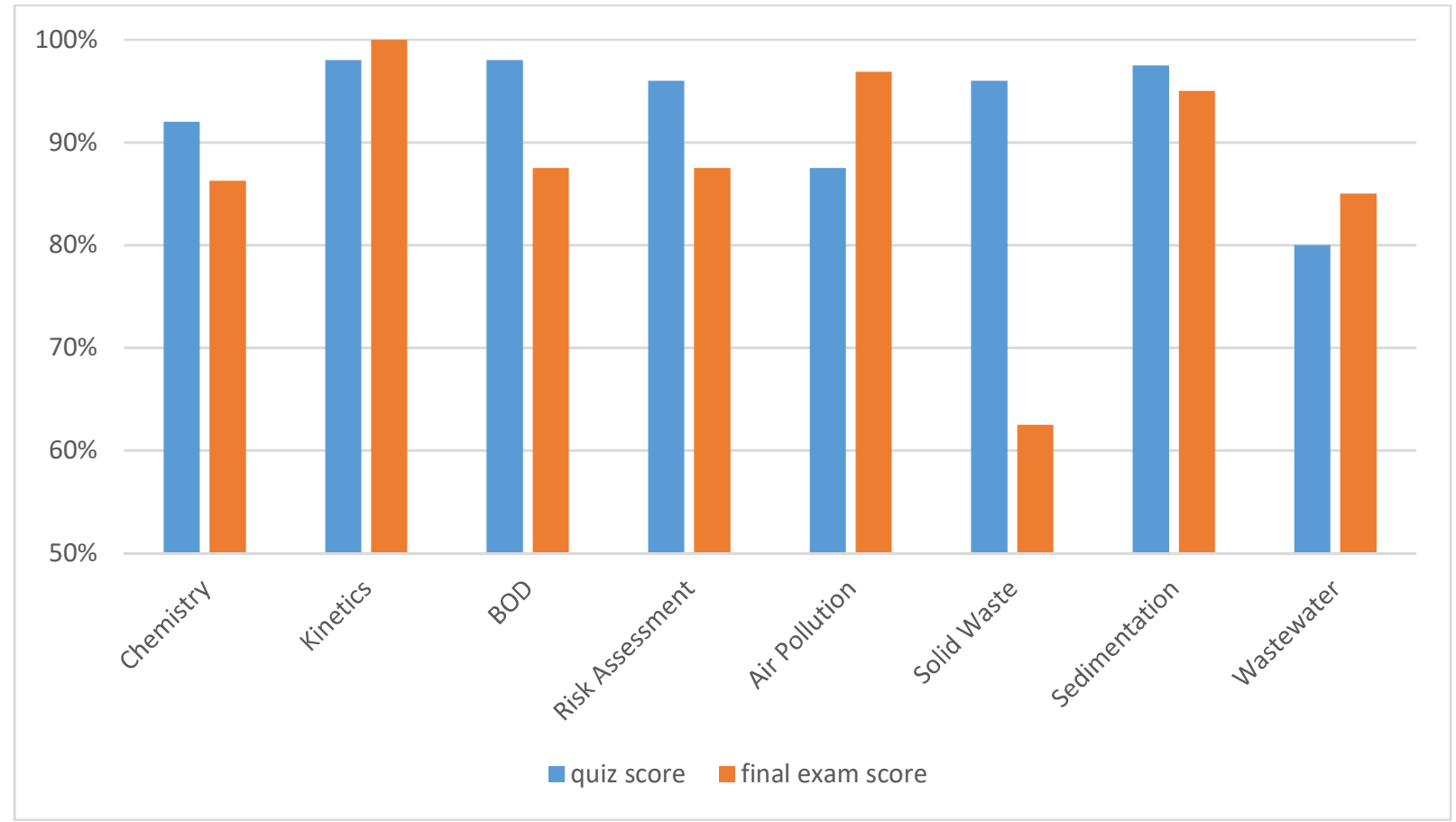

Figure 4 Average quiz and final exam scores 
A survey was administered to receive feedback from the students about the online quizzes. Results are presented below. 14 of the 16 students said they would recommend using online quizzes in the future. Their advantage over homework overall was not as clear, but the feedback is generally positive. Students did observe and appreciate the immediate feedback.

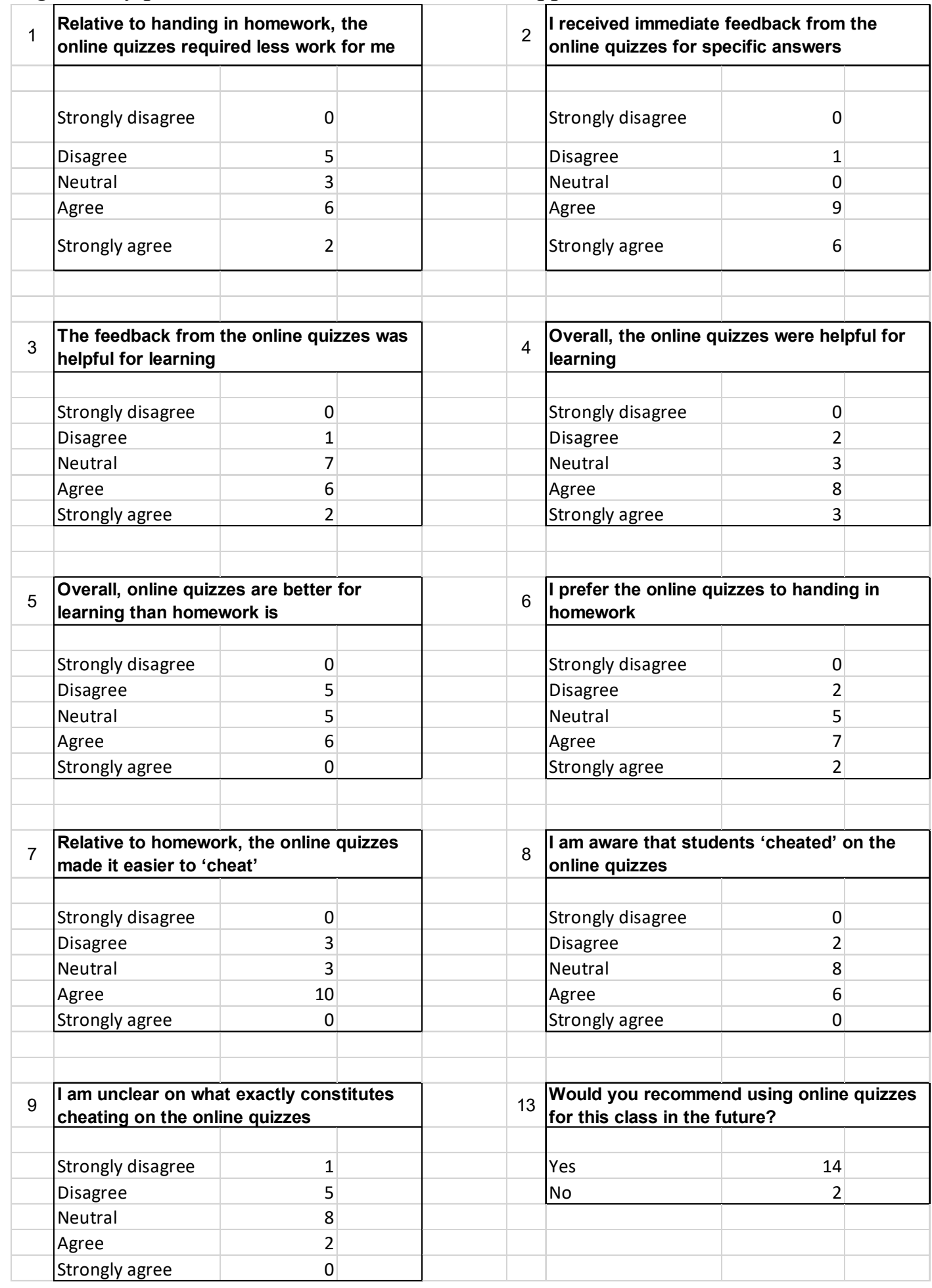

Figure 5. Results of student feedback survey - multiple choice questions 
Q10

What do you see as advantages of the online quizzes?

Answered: 14 Skipped: 2

1. Immediate feedback

2. They are easy because you dont have to hand them in

3. Provide immediate feedback while problems are still fresh in your mind

4. Immediate feedback to answers rather than waiting until they've been graded.

5. I like that I know if I have the correct answer or close to a correct answer, with the multiple choice. I also like the instant feedback.

6. Two tries,

7. More practice on the material, question's we haven't seen in class

8. Immediate feedback as to whether or not answers were correct

9. You know whether or not you did the problem right while it is still fresh in your mind.

10. I don't know

11. Instant feedback. Quick

12. They made tests easier

13. multiple choice easy to keep organized immediate feedback

14. It is easier to complete and you don't have to worry about handing it in during class.

Q11

What do you see as disadvantages of the online quizzes?

Answered: 12 Skipped: 4

1, None

2. $i$ could forget to do them if they are due at a certain time

3. People are able to pass on answers and avoid working through the problems

4. Students are more prone to cheat either by guessing or looking up the answers.

5. A student could potentially just get the answers of another because no work is required to be shown.

6. No partial credit

7. Question's we haven't seen in class so more difficult to know if we are doing them the right way.

8, Less practice

9. Not knowing the questions before hand made it hard, I was scared of not finishing before something computer related kicked me out and being confused

10. No solutions

11. Easy to get answers

12. easy to cheat

Figure 6. Results of student feedback survey - open ended questions 
Q12

Please give any additional comments about the online quizzes

Answered: 5 Skipped: 11

1. None

2. Make sure that there are solutions to the problem after the quiz is over so the person can see how the problem is done

3. I liked them as an alternative

4. Some of the answers were rounded in a way that was difficult to track based on calculations

5. I liked the quizzes, I do believe they were helpful for learning and would recommend in the future. It would be nice when we submit them if there is some more feedback with regards to the process of how to get the answer. Maybe supply some more intermediate answers. Supplying the intermediate answers after submission helped me see where I went wrong in the process. Also, the cheating policy was kind of unclear. Because I would work with a friend and we would fill out the answers together as well, but we both did the proper work to compare answers.

Figure 7. Results of student feedback survey - additional comments

\section{Observations and future direction}

Following are some observations about the switch on online quizzes:

1. With the students working together, quiz scores were high, did not do a good job assessing students individually, and tended to bias the overall grades upward. In the future, the quizzes will count for less in the class and more paper quizzes will be given during the semester for individual assessment.

2. The quizzes, likely due to the immediate feedback, seemed to have eliminated the previously observed low scores on final exam scores related to kinetics and chemistry.

3. Students did not comment about not having graded homework to study from for the exams. They were given a thorough study guide for each exam with solved problems, and this may have served the same role as graded homework.

4. The quality of individual student work in homework cannot be observed in this approach, which is perhaps missing a valuable interaction between instructor and student.

5. What constitutes "cheating" in the quiz approach is unclear, which is why this was addressed in the survey. The survey indicated that the students were unclear on this. More clear direction and rules are needed.

6. A hybrid approach with a few written homework assignments along with the online quizzes may be useful. 


\section{Summary}

Online quizzes in place of homework can be useful tools to provide immediate feedback to students on engineering problems, to offload some of the grading time for classes, and to increase exam scores. The use of such quizzes in an Environmental Engineering class improved the final exam scores of students on two topics that previous classes had trouble with and as a result improved their overall scores. In the format that they were administered, the quizzes were not as useful for individual assessment as written homework was and additional assessments such as in-class quizzes or additional exams should be used.

The students had positive comments on the immediate feedback provided by the quizzes and a strong majority of the students recommended using them in the future. This approach will continue to be used for most, but perhaps not all, of the assignments in Environmental Engineering and may be integrated into other classes as well. 


\section{References}

[1] D.E. Schmidt, D.V.P. Sanchez, and S.J. Dickerson, (2017), “Increasing Student Engagement and Motivation by Replacing Homework with Assignment-Quizzes", Proceedings of the 2017 ASEE Annual Conference, Columbus, Ohio, June 25-28, 2017.

[2] G.C. Brunnhoeffer, G. C. (2017), "Homework Is So 20th Century," Proceedings of the 2017 ASEE Annual Conference \& Exposition, Columbus, Ohio, June 25-28, 2017.

[3] R.J. Dufresne, Mestre, D.M. Hart, and K.A. Rath, "The Effect of Web-Based Homework on Test Performance in Large Enrollment Introductory Physics Courses", Journal of Computers in Mathematics and Science Teaching (2002) 21(3), pp. 229-249. 